\title{
Left Bundle Branch Pacing: Bench to Bedside and beyond
}

\author{
Shunmuga Sundaram ${ }^{1}$, Vanita Arora ${ }^{2}$, Narayanan Namboodiri ${ }^{3}$, Vivek Kumar ${ }^{2}$, Aditya \\ Kapoor $^{4}$, and Pugazhendhi Vijayaraman ${ }^{5}$ \\ ${ }^{1}$ Velammal Medical College Hospital and Research Institute \\ ${ }^{2}$ Max Healthcare \\ ${ }^{3}$ Sree Chitra Tirunal Institute for Medical Sciences and Technology \\ ${ }^{4}$ Sanjay Gandhi Postgraduate Institute of Medical Sciences \\ ${ }^{5}$ Geisinger Heart institute
}

May 14, 2020

\begin{abstract}
Cardiac pacing is the only effective therapy for patients with symptomatic brady-arrhythmia. Traditional right ventricular apical pacing causes electrical and mechanical dyssynchrony resulting in left ventricular dysfunction, recurrent heart failure and atrial arrhythmias. Physiological pacing activates the normal cardiac conduction thereby providing synchronized contraction of ventricles. Though His bundle pacing (HBP) acts as an ideal physiological pacing modality, it is technically challenging and associated with troubleshooting issues during follow up. Left bundle branch pacing (LBBP) has been suggested as an effective alternative to overcome the limitations of $\mathrm{HBP}$ as it provides low and stable pacing threshold, lead stability and correction of distal conduction system disease. This paper will focus on the implantation technique, troubleshooting, clinical implications and a review of published literature of LBBP
\end{abstract}

\section{Hosted file}

LBBP review for JCE.docx available at https://authorea.com/users/309818/articles/451221-leftbundle-branch-pacing-bench-to-bedside-and-beyond 


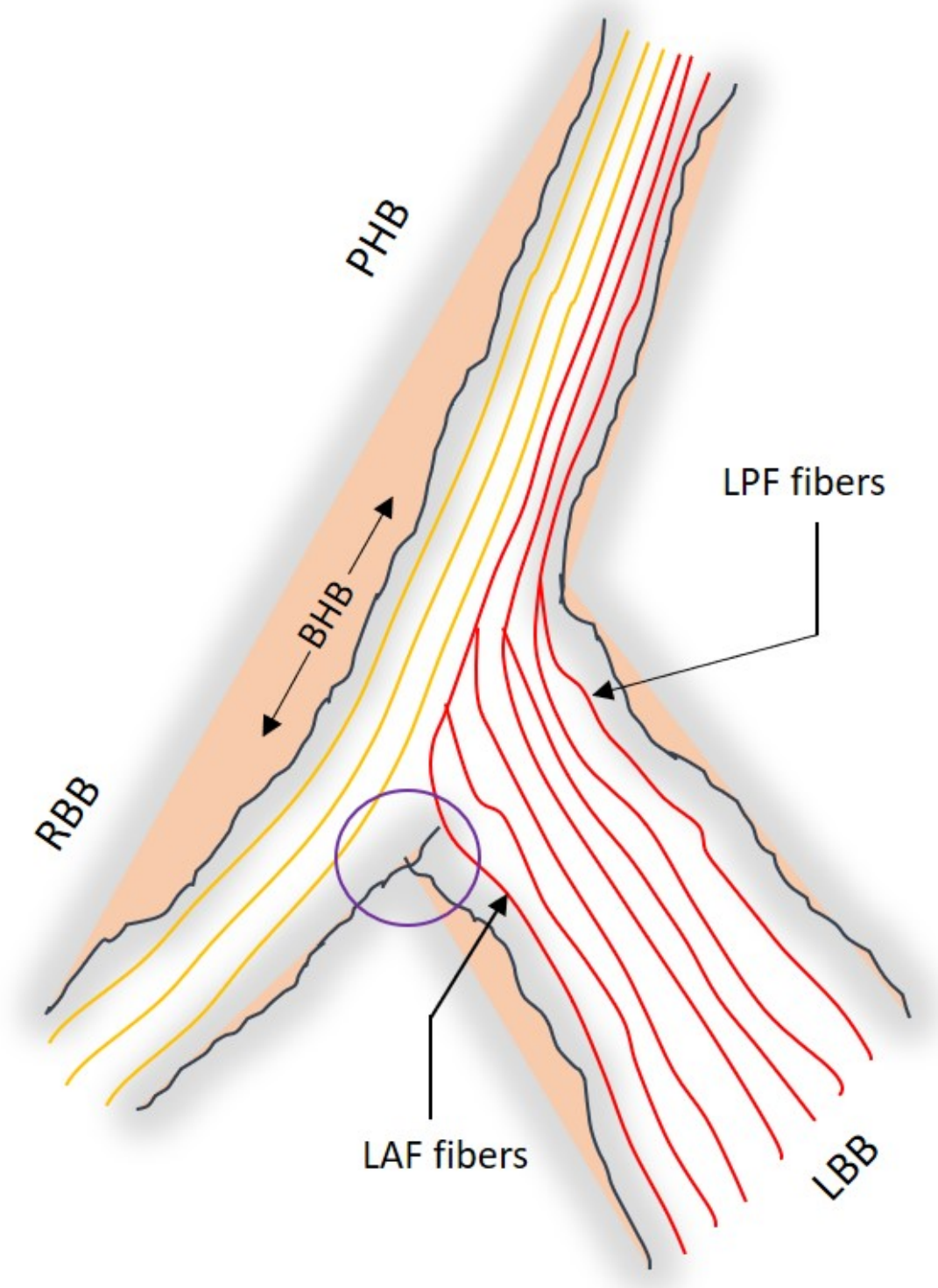



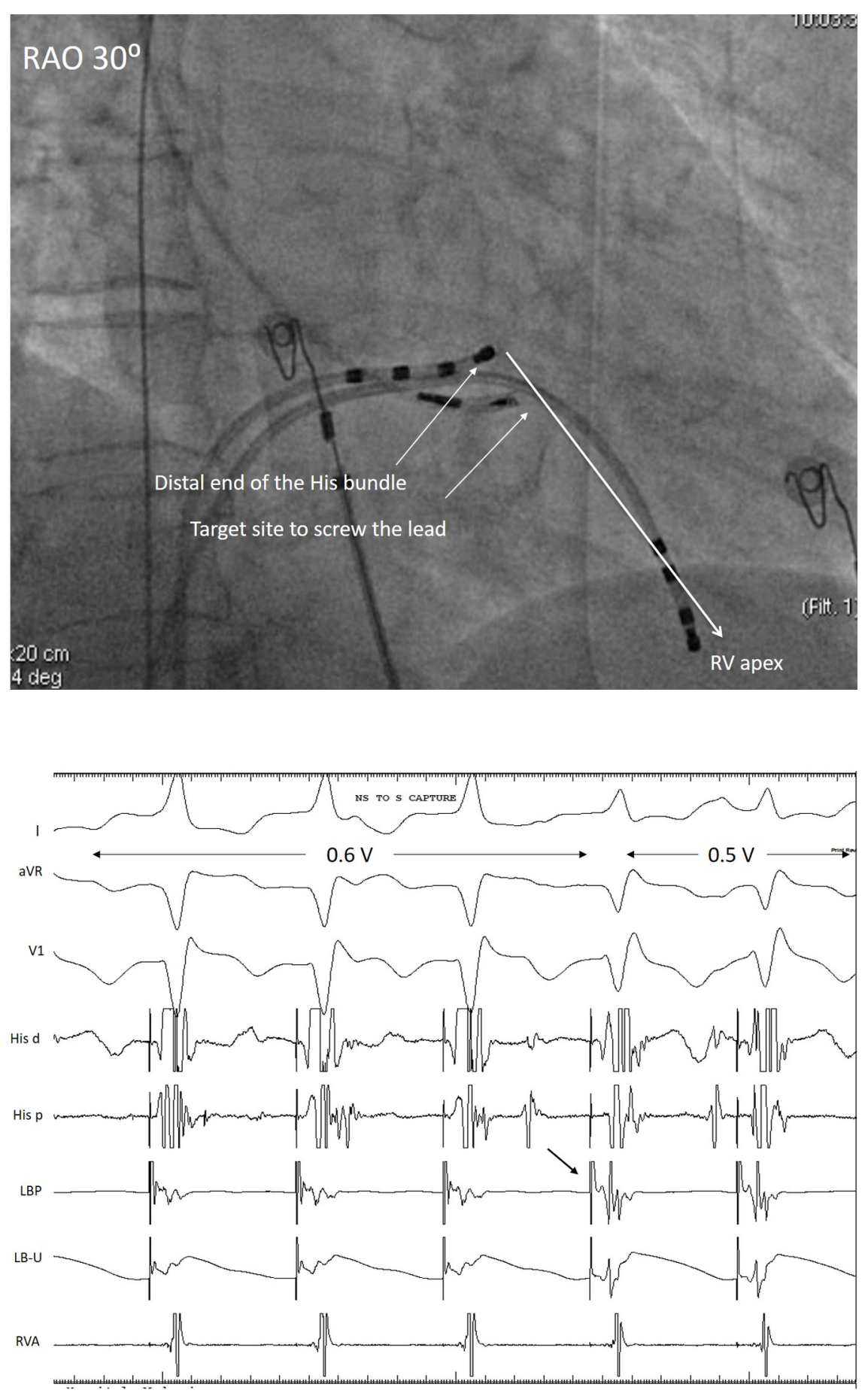

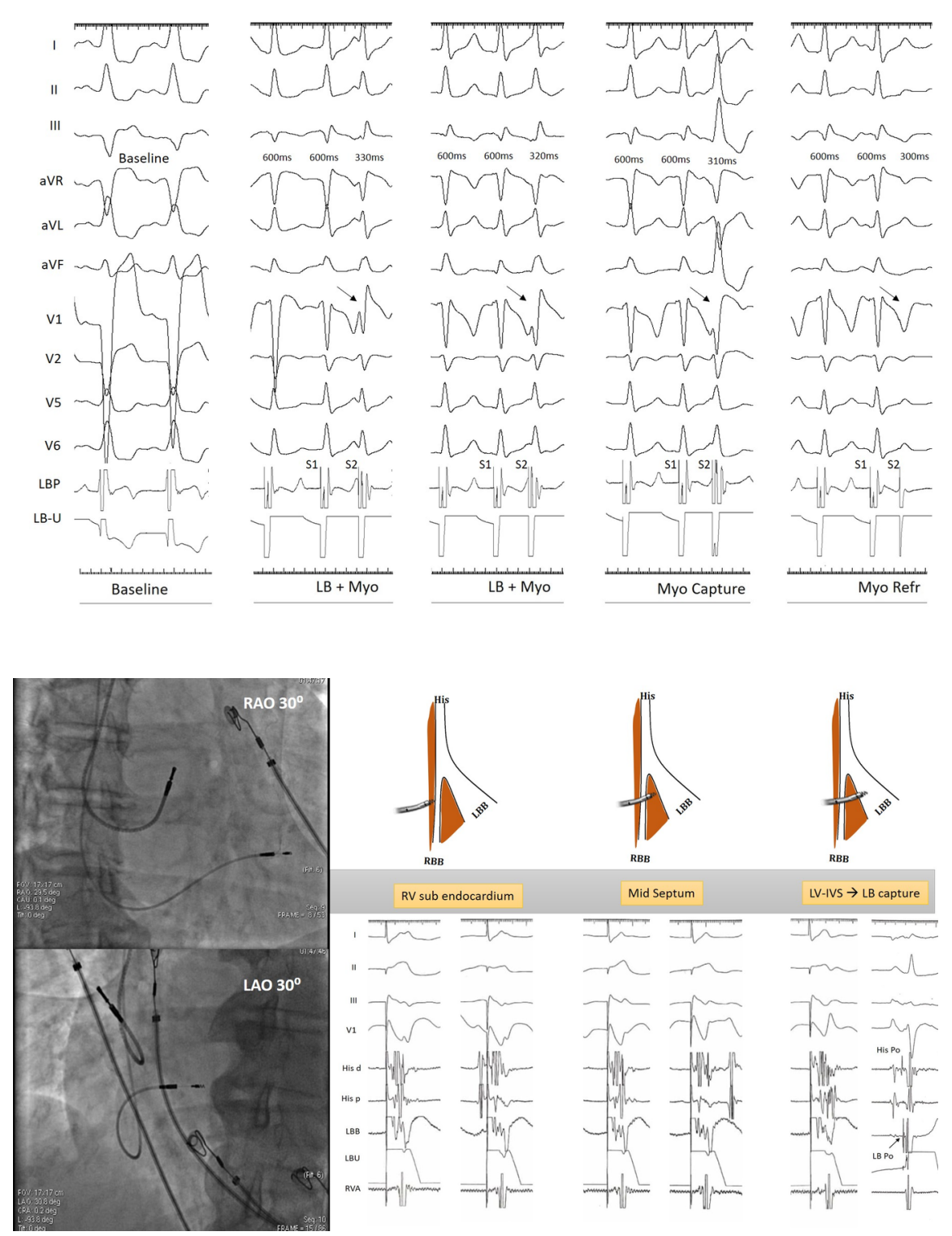

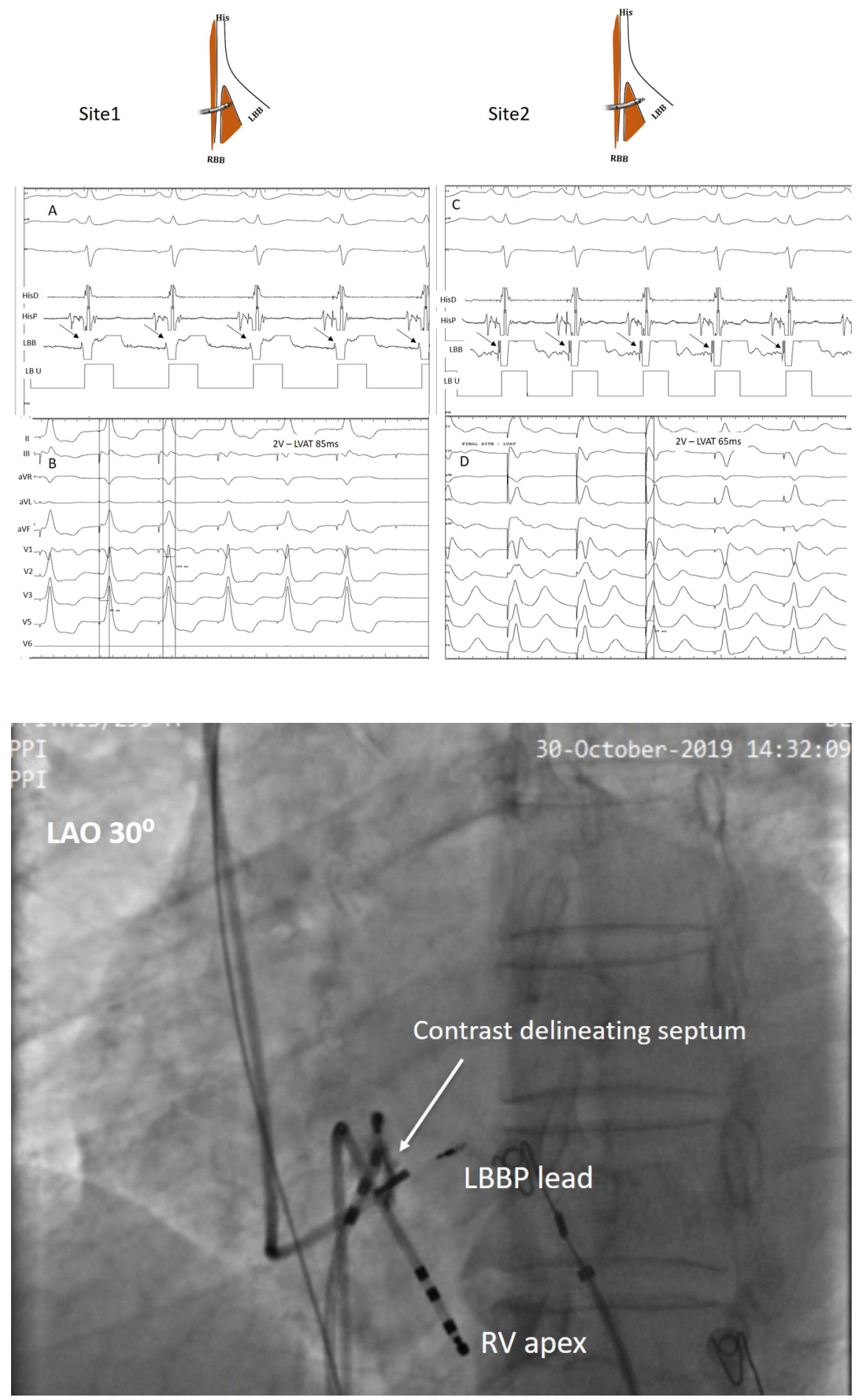

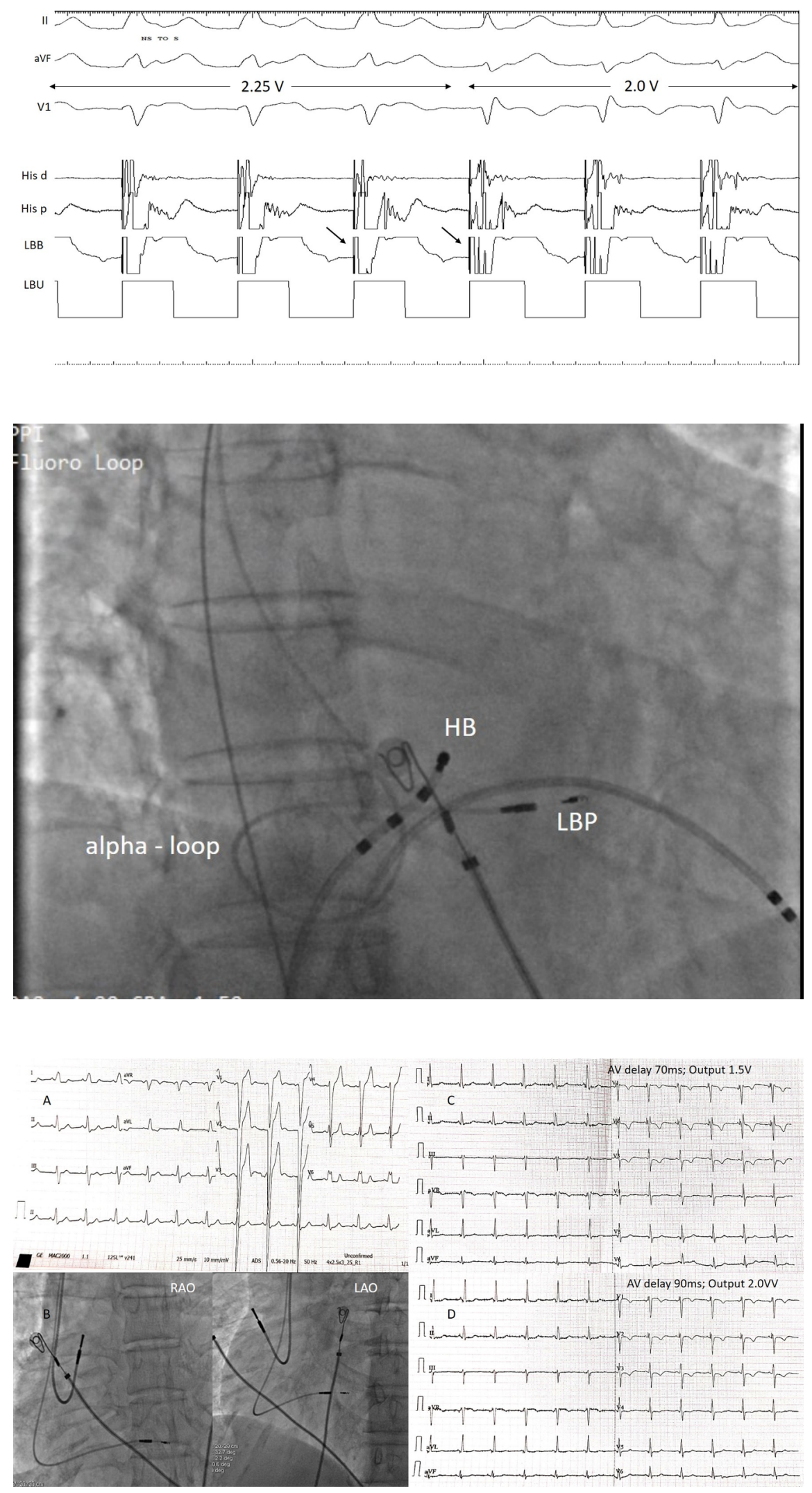


\begin{tabular}{|c|c|c|c|c|c|c|c|c|}
\hline SI. No & Study & $\begin{array}{l}\text { Number of } \\
\text { patients[n] }\end{array}$ & $\begin{array}{l}\text { Implant success } \\
\text { rate[\%] }\end{array}$ & $\begin{array}{c}\text { Paced } \\
\text { QRS[ms] }\end{array}$ & $\begin{array}{l}\text { Threshold } \\
\text { At implant } \\
\text { V@0.5ms }\end{array}$ & $\begin{array}{c}\mathrm{R} \text { wave at } \\
\text { implant[mV] }\end{array}$ & $\begin{array}{l}\text { Lead } \\
\text { revision } \\
\text { rates }\end{array}$ & Objective of the study \\
\hline \multicolumn{9}{|c|}{ LBBP for Bradycardia } \\
\hline 1 & Vijayaraman et al [12] & 100 & $93 \%$ & $136 \pm 17$ & $0.6 \pm 0.4$ & $10 \pm 6 \mathrm{mV}$ & $3 \%(3)$ & $\begin{array}{l}\text { Prospective study in patients requiring } \\
\text { pacing for bradycardia or heart failure } \\
\text { indications }\end{array}$ \\
\hline 2 & Liet a[17] & 87 & $80.5 \%$ & $113.2 \pm 9.9$ & $0.76 \pm 0.22$ & $11.99 \pm 5.36$ & 0 & $\begin{array}{l}\text { Prospective study in patients requiring } \\
\text { pacing for bradycardia indications }\end{array}$ \\
\hline 4 & Hou et al [18] & 56 & NR & $117.8 \pm 11.0$ & $0.5 \pm 0.1$ & $17 \pm 6.7$ & 0 & $\begin{array}{l}\text { Prospective study assessing LV } \\
\text { synchrony in HBP vs LBBP vs RVP }\end{array}$ \\
\hline 5 & Liet al [14] & 33 & $90.9 \%$ & $112.8 \pm 10.9$ & $0.76 \pm 0.26$ & 14.4 & $3.03(1)$ & Prospective study of LBBP in AV block \\
\hline 6 & Zhang et al[16] & 23 & $95 \%$ & $112.6 \pm 12.14$ & $0.68 \pm 0.2$ & $9.28 \pm 5.00$ & 0 & $\begin{array}{l}\text { Prospective comparative study of LBBP } \\
\text { over RVP in } 44 \text { consecutive patients }\end{array}$ \\
\hline 7 & Hasumi et al [25] & 21 & $81 \%$ & $116 \pm 8.3$ & $0.77 \pm 0.07$ & $9.1 \pm 1.4$ & 0 & $\begin{array}{l}\text { Retrospective study assessed the } \\
\text { feasibility of LBBP in failed HBP for AV } \\
\text { block }\end{array}$ \\
\hline 8 & Chen et al [26] & 20 & NR & $111.8 \pm 10.7$ & $0.73 \pm 0.2$ & NR & 0 & $\begin{array}{l}\text { Prospective study to compare the } \\
\text { feasibility and ECG patterns during } \\
\text { LBBP vs RVP }\end{array}$ \\
\hline 9 & Jastrzebski et al [10] & 143 & NR & $111.9 \pm 15.1$ & $0.6 \pm 0.3$ & $9.0 \pm 5.1$ & NR & $\begin{array}{l}\text { Prospective study to analyze the } \\
\text { programmed deep septal stimulation in } \\
\text { regard to diagnosis of LBB capture }\end{array}$ \\
\hline 10 & Suet al [27] & 115 & NR & $111.4 \pm 10.3$ & $0.6 \pm 0.2$ & $11.3 \pm 5.4$ & 0 & $\begin{array}{l}\text { Retrospective study to assess LB } \\
\text { current of injury in LBBP }\end{array}$ \\
\hline 11 & Cai et al (23) & 40 & $90 \%$ & $101 \pm 8.7$ & $0.49 \pm 0.22$ & $11.7 \pm 5.3$ & 0 & $\begin{array}{l}\text { Prospective study to assess the cardiac } \\
\text { synchrony in } 5 S \text { patients undergoing } \\
\text { LBBP VS RVP }\end{array}$ \\
\hline 12 & Wang et al [22] & 66 & $94 \%$ & $121.4 \pm 9.8$ & $0.94 \pm 0.21$ & $12.1 \pm 3.6$ & $4.5 \%(3)$ & $\begin{array}{l}\text { Prospective randomized study to } \\
\text { compare the depolarization and } \\
\text { repolarization measures between LBBP } \\
\text { Vs RVP }\end{array}$ \\
\hline 13 & Vijayaraman et al (24) & 28 & $93 \%$ & $125 \pm 15$ & $0.64 \pm 0.3$ & $14 \pm 8$ & 0 & $\begin{array}{l}\text { Retrospective study to assess the } \\
\text { feasibility of HPCSP pacing after TAVR } \\
\text { (LBBP and HBP) }\end{array}$ \\
\hline \multicolumn{9}{|c|}{ LBBP for CRT } \\
\hline 1 & Zhang et al[19] & 11 & NR & $\begin{array}{c}129.09 \pm \\
15.9\end{array}$ & $0.83 \pm 0.16$ & $9.1 \pm 3.4$ & 0 & $\begin{array}{l}\text { Study assessing clinical outcomes of } \\
\text { LBBP in patients with HF, reduced } \\
\text { LVEF and LBBB }\end{array}$ \\
\hline 2 & Wang et al [28] & 8 & $94.5 \%$ & NR & $0.79 \pm 0.18$ & NR & NR & $\begin{array}{l}\text { Retrospective study assessed the } \\
\text { efficacy of } \mathrm{HPCSP}+\mathrm{AVJ} \text { ablation in } \\
\text { patients with AF and ICD }\end{array}$ \\
\hline 3 & Huang et al [15] & 63 & $97 \%$ & $118 \pm 12$ & $0.5 \pm 0.15$ & $11.1 \pm 4.9$ & 0 & $\begin{array}{l}\text { Prospective study to assess the } \\
\text { feasibility and efficacy of LBBP in LBBB } \\
\text { with NICM }\end{array}$ \\
\hline 4 & Wu et al [29] & 32 & $100 \%$ & $110.8 \pm 11.1$ & $0.49 \pm 0.13$ & $11.2 \pm 5.1$ & 0 & $\begin{array}{l}\text { Prospective study to compare CRT } \\
\text { efficacy of } L B B P, H B P \text { and Biv pacing. }\end{array}$ \\
\hline 5 & Jiang et al (21) & $73(63+10)$ & $\begin{array}{l}\text { Atypical BBB-30\% (3) } \\
\text { Typical B8B-82.5\% (52) } \\
75.3 \%\end{array}$ & $\begin{array}{l}133 \pm 14 \\
118 \pm 14\end{array}$ & $0.6 \pm 0.2$ & $13.6 \pm 7.6$ & 0 & $\begin{array}{l}\text { Retrospective study to assess whether } \\
\text { typical use of strict criteria to define } \\
\text { BBB predicts LBBP success }\end{array}$ \\
\hline \multirow[t]{2}{*}{6} & Vijayaraman et al [30) & 325 & $85 \%$ & $137 \pm 22$ & $0.6 \pm 0.3$ & $10.6 \pm 6$ & $2.5 \%(7)$ & $\begin{array}{l}\text { Retrospective study assessed the } \\
\text { feasibilityand outcomes of LBBP in CRT } \\
\text { eligible patients }\end{array}$ \\
\hline & Total & 1244 & $90.65 \%$ & 117.9 & 0.626 & 11.58 & $\begin{array}{l}1.28 \% \\
(14)\end{array}$ & \\
\hline
\end{tabular}

